\title{
Asynchronous Telemedicine Diagnosis of Musculoskeletal Injuries through a Prototype Interface in Virtual Reality Environment
}

\author{
Soheeb Khan ${ }^{1}$, Vassilis Charissis ${ }^{1}$, David Harrison ${ }^{1}$, Sophia Sakellariou ${ }^{2}$, \\ and Warren Chan ${ }^{1}$ \\ ${ }^{1}$ Glasgow Caledonian University \\ School of Engineering and Built Environment, \\ Department of Computer, Communications and Interactive Systems, \\ Glasgow, UK \\ ${ }^{2}$ Royal Infirmary, \\ Department of Radiology, \\ Glasgow, UK \\ soheebkhan@googlemail.com
}

\begin{abstract}
Telehealth provides a much needed option for remote diagnosis and monitoring of various pathologies and patients. Remote provision of health care can offer a two fold support for the medical system and the patients. Primarily it could serve isolated locations and secondly it could monitor a large number of outpatient cases directly on their homes instead of the hospital premises. However in specific cases direct communication and visual data acquisition can be a major obstacle. To this end we have developed a prototype system that could enable the medical practitioners to have real-time diagnosis through 3D captured visual and motion data. This data are recreated in a Virtual Reality environment in the hospital facilities offering a unique system for remote diagnosis. This paper presents the design considerations and development process of the system and discusses the preliminary results from the system evaluation. The paper concludes with a tentative plan of future work which aims to offer the medical practitioners and the patient with a complete interface which can acquire gait data and thus analyse a large variety of musculoskeletal pathologies.
\end{abstract}

Keywords: Virtual Reality, HCI, 3D Visualization, Asynchronous Diagnosis, Telemedicine, Motion Capture.

\section{Introduction}

The demand and utilisation of telemedicine-based care is on the increase due to the highly amplified number of populations striving towards country life and remote living locations. Additionally the financial climate, limited resources and the constant growing population around the globe have prompted an interest from various governing bodies to reform and seek alternative methods for delivering high quality health care [1]. The technological advancements and the increase of communication innova- 
tions have made telemedicine a promising solution for many issues faced by the current health care systems [2, 3]. Patients with musculoskeletal (MSK) issues and injuries comprise the largest number of presentations to General Practitioners (GPs), and continue to require input even post surgical intervention or during the rehabilitation process [4]. Yet the gait analysis of each patient is time-consuming and costly if the patients are located away from the medical and city centres.

The technological innovations in motion-capture (Mo-Cap) systems have made it possible to acquire and collect complex motion data for the biomechanics of musculoskeletal (MSK) structures. Mo-Cap techniques are being deployed and implemented across a range of disciplines. In the past, Mo-Cap setups have been expensive ventures, as they required specialised costly equipment, professional setup, training and allocation of a dedicated large space. This has limited the amount of gait analysis and MSK diagnosis laboratories confining them to cities and highly populated areas. Such laboratories are most commonly situated at a designated facility which requires the patients to travel to and from routinely. Due to the limitation of such facilities, people living in isolated and rural areas have limited or no access to this triage. Markerless motion capture techniques are new and only in the last decade the technology has surfaced as a useable product for the mass consumers. Contemporary technological breakthroughs, related to cameras, projectors and videogames fuelled the development of cost-efficient, consumer-based peripherals such as the Microsoft "Kinect" that could be utilised off-the-shelf for a fraction of the typical Mo-Cap suites [5]. Despite the significantly reduced price, only a minor impact to the final quality of the derived motion data is noted, the latter not constituting a significant drawback for the majority of the applications.

The current capacity in computer processing and accessibility of variety of 3D development packages has enabled visualisation of complex anatomy in real-time virtual environments. Mass developments of videogames, virtual reality (VR) and 3D programs have made possible for 3D engines to be utilised across various industries as common platforms for real-time visualisation purposes. This has prompted medical information to be presented in a much-improved manner by the use of photorealistic 3D models and user-friendly interfaces.

The main objective of our proposed system is to employ inexpensive hardware such as the 'Kinect' in conjunction with 3D VR environments, and a user-friendly interface to obtain locomotion data that can be of diagnostic value for clinicians. The proposed work presents an asynchronous Telemedicine based Musculoskeletal Diagnosis Service, which enables the health professionals to asynchronously perform gait analysis and diagnosis. The telemedicine based system utilises 3D medical visualisation, Virtual Reality and Motion Capture systems. Furthermore the system supports active learning and development for healthcare professionals through the use of a user-friendly interface and appropriately designed Human Computer Interaction (HCI) in conjunction with photorealistic 3D medical visualisation. The system was evaluated both with qualitative and quantitative methods by ten medical practitioners offering promising results with regards to the HCI usability, and data manipulation through online real-time communication. The initial trials utilised a default MSK 3D 
human model which was attached to various motion capture data produced in realtime in a laboratory environment and transmitted to the medical group's location.

\section{Innovative Motion Capturing Solutions}

Contemporary technological breakthroughs, related to the videogames industry and associated peripheral devices have fuelled researchers to begin development of costefficient, home based telemedicine systems. Innovative inexpensive optical sensor technology designed to enhance the video game experiences have become an easy obtainable solution for multiple complex issues and desires for human computer interaction (HCI) [6,7]. Clinicians and researchers have begun to experiment and develop tools with such devices to assist motion driven tasks $[8,9,10]$. A sufficient amount of interest has been to use such tools as an aid for rehabilitation purposes [10,11]. Attygalle developed a tool for a home based rehabilitation setup, which incorporated the standard Biofeedback rehabilitation program. Using a Nintendo Wii remote, Attygalle successfully recorded data concerning rehabilitation for stroke patients. The data was asynchronously analysed by therapists to track the patient's progression over time.

Concurrent studies developed a system that utilised the Kinect's hand gesture capabilities to be employed in live surgery [11]. This system facilitated health professionals in a sterile environment to navigate, manipulate and control MRI and CT images through Natural User Interface (NUI). The system proposed by Zöllner successfully employed the Kinect as a navigation tool for the visually impaired [12]. Similarly there are many projects that are currently experimenting with this technology in various other disciplines [10,11,12]. Other studies demonstrated Kinect's tracking capabilities which delivered moderate results when compared to the Vicon system $[13,14]$.

IPI Soft is a motion capturing software which utilises such videogame peripherals to achieve low budget motion tracking. Various other tools are available that also utilise this technology for tracking purposes but in comparison IPI Soft excels. Although used mostly by the entertainment industry, this research showed that this tool has not yet been utilised for gait analysis. It is yet to be demonstrated if such devices and software could be utilised to obtain data that can be of diagnostic value to the clinician and comparative studies of its performance against the traditional marker based motion capture system (vicon).

\section{System Rationale}

The proposed system design rationale consists of three phases: Data Collection, Data Processing and Diagnosis. Phase one consists of gathering and submitting the essential data required for motion analysis and clinical musculoskeletal diagnosis. The proposed system requires this process to be carried out remotely and independently by patients or health professionals. 
The submitted data will be transmitted to a designated processing centre. Phase 2 requires the data to be processed into a viewable and readable medium for the health professional. Phase 3 enables the specialist/ health professionals to view and analyse the data to make a diagnosis. Further courses of action could be delivered to the patient via email or phone. These phases will be asynchronous and independent from one another, which will not require any instant reaction or live contact between them. A detailed description of each phase is illustrated in the architecture of the proposed system. See Fig 1.

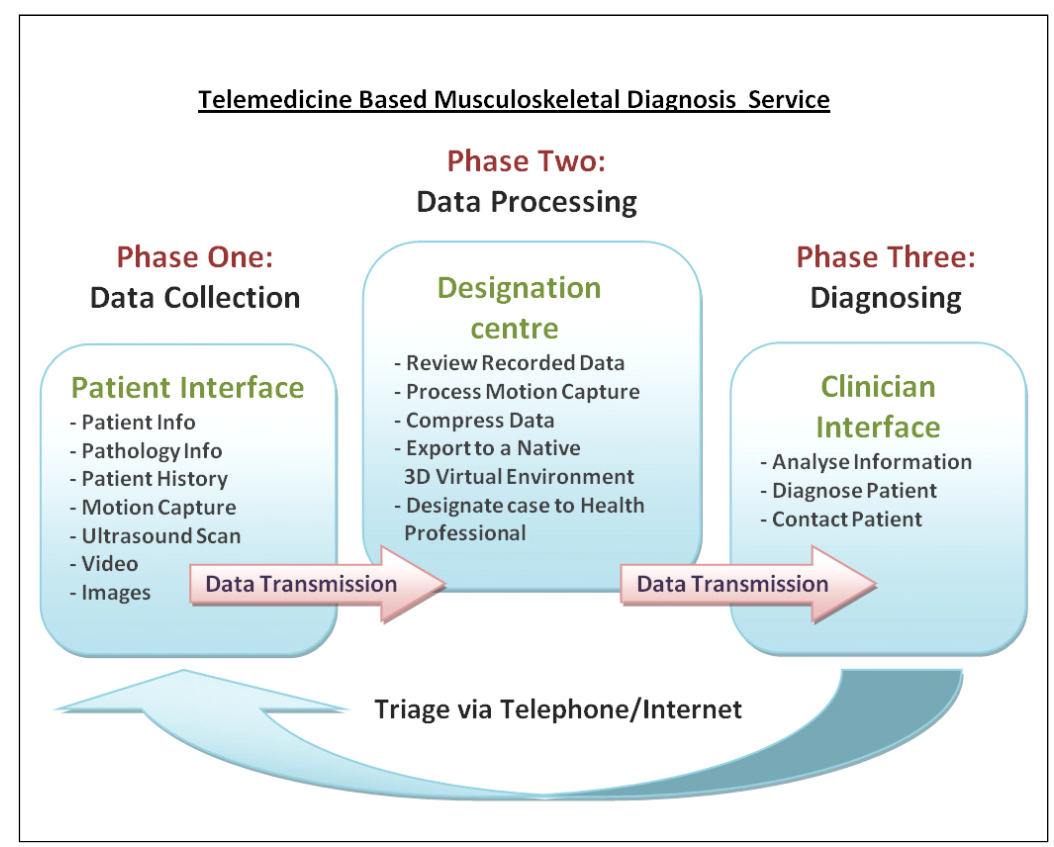

Fig. 1. Architecture of the Proposed System

\section{Comparative Analysis of Optical Sensors}

A thorough analysis of existing off-the-shelf sensor equipment was deemed necessary so as to define the best possible combination of hardware. As software compatibility is a major issue with all new technologies analysed, we concluded that the best option currently is somehow dictated by the designated software used for multiple tracking sensors, namely ipiSoft. Consequently, the peripherals that were taken in consideration were chosen as compatible with the specific software and utilised by the ipi Desktop motion capture system. 
The three main systems that are most compatible with ipiSoft are Microsoft Kinect Sensor, Asus Xtion sensor and PlayStation Eye Digital camera. By analysing the user guides for each of the systems provided by ipiSoft, the different optical sensors in regards to the proposed work were compared. The systems were compared on price, portability, calibration, space utilisation, complexity and time of setup as well as the size and accuracy of the recorded data. This allowed the selection of the most appropriate kit for the proposed system. The paper furthermore presents an analysis of benefits and drawbacks of three different setups recommended by ipiSoft in relation to the proposed system as presented in Table 1 below.

Table 1. Affordable Optical Sensors Comparison

\begin{tabular}{|c|c|c|}
\hline Optical Sensors & Positive & Negatives \\
\hline $\begin{array}{l}\text { Microsoft Kinect } \\
\text { (Available from } \\
\text { £99 per unit) }\end{array}$ & 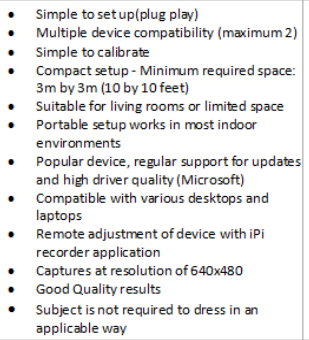 & $\begin{array}{l}\text { - Prone to Occlusion Problem } \\
\text { whilst using single device } \\
\text { - Require ACDC power supply } \\
\text { - } \quad \text { Mly } 30 \text { fps frame rate } \\
\text { feximum capture volume7 by } 7 \\
\text { - Only } 30^{\circ} \text { of coverage while using } \\
\text { multiple devices } \\
\text { - } \quad \text { require systems with multiple } \\
\text { USB controllers }\end{array}$ \\
\hline $\begin{array}{l}\text { ASUS Xtion } \\
\text { (Available from } \\
£ 130 \text { per unit) }\end{array}$ & $\begin{array}{l}\text { - Simple to set up(plug play)Multiple Device } \\
\text { compatibility } \\
\text { - Compact setup - Minimum required space: } \\
3 \mathrm{~m} \text { by } 3 \mathrm{~m} \text { ( } 10 \text { by } 10 \text { feet } \\
\text { - Does not require power supply except USB } \\
\text { - Allow } 60 \text { fps frame rate (at } 320 \times 240 \\
\text { resolution only) } \\
\text { - Decent Quality Results } \\
\text { - Subject is not required to dress in an } \\
\text { applicable way }\end{array}$ & $\begin{array}{l}\text { - Recently introduced (prone to } \\
\text { errors) } \\
\text { - Less popular device with no } \\
\text { regular updates and lower drivers } \\
\text { quality } \\
\text { - limited compatibility to certain } \\
\text { desktops and laptops } \\
\text { - positioning and adjustment, only } \\
\text { manual } \\
\text { - Maximum capture volume7 by } 7 \\
\text { feet (approx. } 2 \text { by } 2 \text { meters) } \\
\text { - The More Expensive Device }\end{array}$ \\
\hline $\begin{array}{l}\text { PlayStation Eye } \\
\text { Camera(Available } \\
\text { from } £ 15 \text { per } \\
\text { unit) }\end{array}$ & 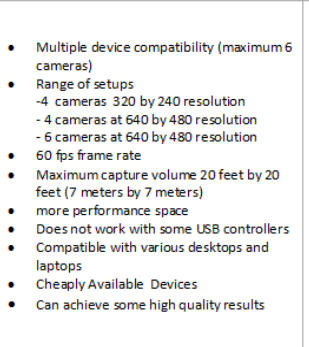 & $\begin{array}{l}\text { - Complicated to set up and } \\
\text { calibrates } \\
\text { - Needs large space, minimum } \\
\text { space required. } 4 \text { meters by } 4 \\
\text { meters } \\
\text { - additional tripods are required } \\
\text { for an effective setup } \\
\text { - Subject is required to wear } \\
\text { certain clothing } \\
\text { - A powerful computing system is } \\
\text { - Lequired to record the data } \\
\text { - Canited Portability } \\
\text { - not suitable for compact or living } \\
\text { - roomenvironments } \\
\text { require systems with multiple } \\
\text { - No controllers } \\
\text { No motor, allows only manual } \\
\text { positioning }\end{array}$ \\
\hline
\end{tabular}

After analysing the gathered information the most appropriate optical sensor for the proposed system is the Microsoft Kinect. In comparison to the 'ASUS Xtione', the 'Kinect' is the observable choice. Both of the depth cameras are very alike in technology and deliver similar results. The Kinect excels as the more popular device due to the regular updates and high quality driver provided by Microsoft. The Kinect is compatible with most laptops and desktops which has prompted both professionals and enthusiasts to develop features using this device to be utilised for a variety of 
purposes. These features could potentially be incorporated and utilised by the proposed system. In contrast ASUS Xtione is less popular and is only available to purchase from specific shops. The system driver's and updates are not as frequent as the updates provided by Microsoft. This could make the setup unstable and prone to error. ASUS Xtione is unsuitable due to its limitation and restrictions in compatibility as it works only with certain laptops and desktops. The Asus device allows recording at 60 frames per second (fps) in comparison to the 30fps provided by the Kinect, this is only possible though when recording at a capture resolution of $320 \times 240$. Both devices record at $30 \mathrm{fps}$ when recording at capture resolution of $640 \times 480$. Although the recorded frame rate is faster at a lower resolution this has an effect on the quality of the data which will eventually be used to track the motion resulting in less accurate results and thus negating the advantage of a higher frame rate.

Although the six-camera based PlayStation Eye setup has been suggested as the recommended choice by ipiSoft for the best results, there are certain limitations and attributes that make this setup less applicable and practical for the proposed system. As it is essential to utilise minimum 3 PlayStation Eye cameras for any motion tracking data, this results in a complex, time consuming installation requiring significant fine tuning. There are various procedures and tasks that need to be carried out in order to obtain functional and efficient calibration. The calibration process is prone to errors and requires the user to patiently adjust and calibrate the cameras until the desired results are achieved. In a remote location, however, the system would need to follow a fast and errors free installation. Maintenance is also required to be minimum with easily accessible and regular software and hardware updates.

Portability is also essential as the proposed system would require a remote setup which will enable the user to capture their movement independently from their homes or remote facilities. As mentioned before, the proposed system is also targeted at people who may have limited or no knowledge of using computers. Calibration and installation of the setup needs to be very simple so that it could be carried out independently without having to carry out complicated procedures. Comparatively to the PlayStation Eye setup, the Kinect setup is substantially easier to install and does not require any additional equipment for recording other than the laptop and cables. The minimum required space for the Kinect setup is $3 \mathrm{~m}$ by $3 \mathrm{~m}$ (10 by 10 feet) which is ideal for environments such as living rooms and facilities with limited space capacity. This setup is portable as it does not require special calibration unlike the Sony PlayStation Eye setup.

Overall the three tested systems were further compared with other different Mo-Cap technologies used in prior research studies [15,16]. Particular focus was given to alternative inexpensive but effective solutions. These included the optical sensors predominantly utilised for video gaming purposes and Mocap tool's like ipiSoft that are utilised for low budget projects. By experimenting with these alternative solutions the best device for the proposed system was chosen as presented above. 


\section{$5 \quad$ Evaluation}

Based on the aforementioned observation our system entailed 2 Kinects and a laptop in order to retrieve the motion data and compile them with the use of ipiSoft.

The three-dimensional visualisation was further exported and combined through Unity 3D to a default three-dimensional model of a human body. The latter serves the purpose of presenting the potential injury location rather than presenting a detailed model of the patient.

The system was evaluated with 10 users in the Virtual Reality and Simulation laboratory (VRS lab) of Glasgow Caledonian University (Glasgow, UK). The laboratory is equipped with state of the art $\mathrm{HD} / 3 \mathrm{D}$ projector, 65inch $3 \mathrm{DTV}$, and surround audio equipment. The aforementioned devices run through a set of custom-built workstations powered by dual Hexacore Xeon CPUs and NVidia Quadro 4000 Fermi graphics cards.

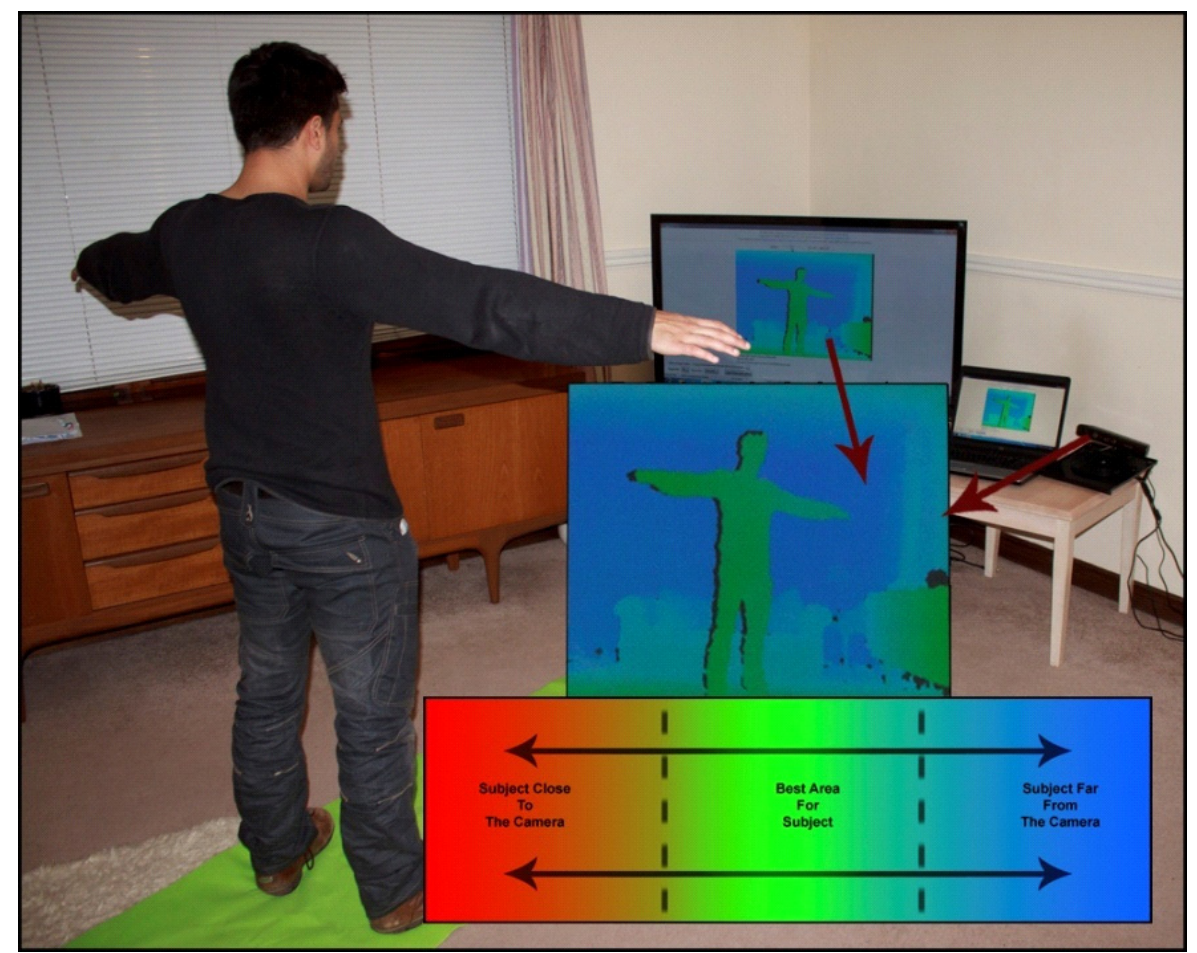

Fig. 2. The subject is being recorded using a Kinect sensor

Data from this experiment was successfully retrieved which was processed and mapped on to a 3D model to replicate the movement in to a virtual environment. This process has been demonstrated in Fig 3. Further work is required to determine if the retrieved data could be utilised for gait analysis and MSK diagnosis. 


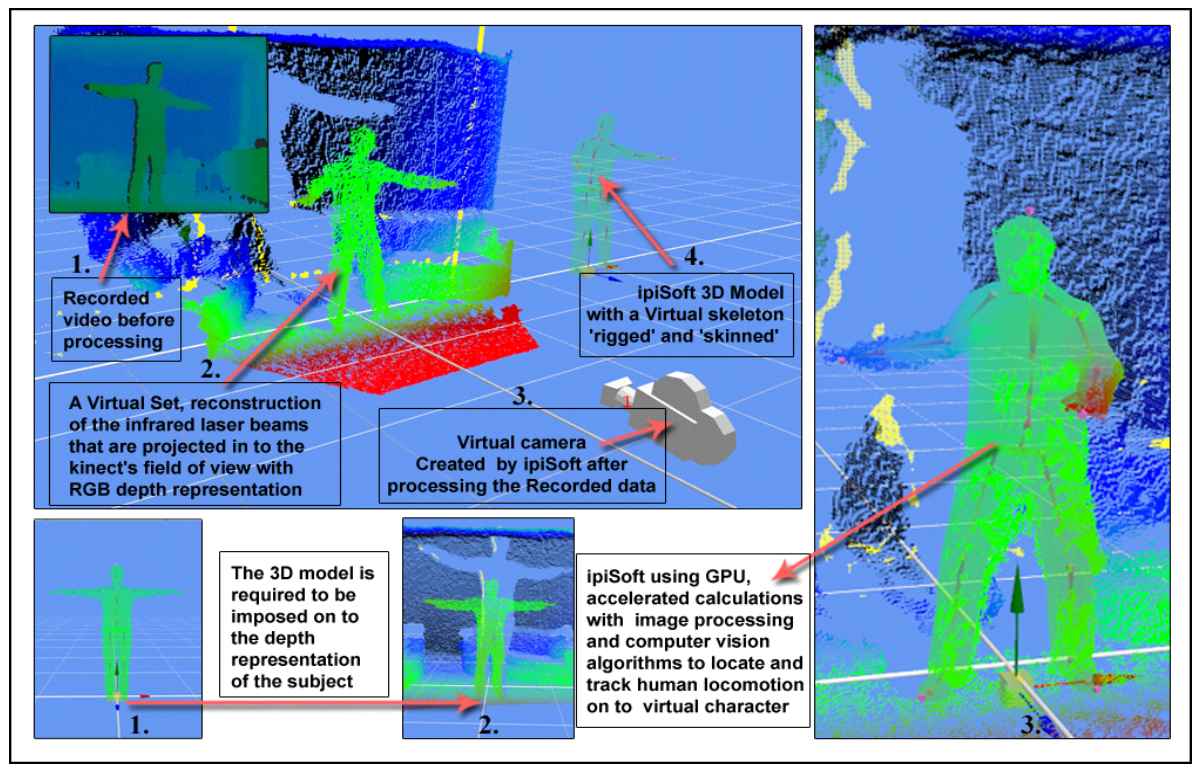

Fig. 3. Motion mapping data on to the virtual character in ipiStudio

\section{$6 \quad$ Results and Discussion}

Through the initial trials the system performs sufficiently in order to capture and transmit motion information for a preliminary diagnostic appraisal. This observation was unanimously noted by the participants. As this is the intention of the proposed system the rough estimation of movement offers clear information that could assist a medical practitioner to estimate the musculoskeletal injury in a long distance surgery. In turn the medical doctor can provide a therapy and through the specific system can monitor the progress of the patient remotely. Some users suggested also that such system could extend its usage from the remote locations to even the typical follow up sessions in order to minimize the crowding effect of visiting patients in the hospital premises.

The system's main drawback is the quality of the derived data that could not be used, in this raw format directly, for precise gait analysis. Such action requires very specific measurements, which need to be captured by multiple very precise cameras and through an iterative process of marking different sections of the leg, foot and ankle. Interestingly the complete gait analysis of data is also falling within the asynchronous process and as such the detailed information need further exploration before any patient consultation or treatment. The precision required in this case affects significantly the cost of the related hardware and software that is required to capture the data. Furthermore complicated and time-consuming calibration and maintenance as well as specialist operators are other major issues for deploying such services in multiple points across the country and particularly in remote locations. 
On our proposed system we are particularly keen to increase quality to acceptable levels for generic diagnosis and monitoring as the required equipment is accessible, cost effective and simple to run and maintain. The users during the trial commented in favour of this approach. The required time of set up of the system was within a 5 minutes margin and the data transmission was in all the trials well within an hour (without any major data optimization).

\section{Conclusions}

This paper presented the development process and system rationale of our proposed, prototype telemedicine system. The particular system is designed for remote diagnosis of musculoskeletal injuries and monitoring patient rehabilitation through gait analysis motion capture. The main objective of the system if to offer a clear, simple and cost effective method for both medics and patients. As such the system employs a variety of off-the-shelf equipment and a custom made software application captures and visualise the information for both groups through a virtual environment. The initial trials presented successful transfer and visualisation of motion capture and anatomical information.

Our tentative plan for future work will aim to identify the potential accuracy issues in comparison to a full motion capture system designed for precise gait analysis which is currently used by the medical and allied health practitioners. Additional information might be incorporated and explored through simplified ultrasound data acquisition. Finally we aim to install the system in satellite GP practises in remote locations across Scotland and evaluate the speed, usability and efficiency of the system in real life conditions.

\section{References}

1. Bower, P., Cartwright, M., Hirani, S., Barlow, J., Hendy, J., Knapp, M., Henderson, C., Rogers, A., Sanders, C., Bardsley, M., Steventon, A., Fitzpatrick, R., Doll, H., Newman, S.: A comprehensive evaluation of the impact of telemonitoring in patients with long-term conditions and social care needs: protocol for the whole systems demonstrator cluster randomised trial. Studies in BMC Health Services Research 11, 184 (2011)

2. Blank, E., Lappan, C., Belmont, P.J., Machen, M.S., Ficke, J., Pope, R., Owens, B.D.: Early Analysis of the United States Army's Telemedicine Orthopaedic Consultation Program. Studies in Journal of Surgical Orthopaedic Advances 20, 50-55 (2011)

3. Syst Kuo, C.H., Liu, L.: Development of a Web-Based Telemedicine System for Remote ENT Diagnoses. In: Studies in 2010 IEEE International Conference on System Science and Engineering (ICSSE), pp. 565-570 (2010)

4. Sakellariou, S., Charissis, V., Grant, S., Turner, J., Kelly, D., Christomanos, C.: Virtual Reality as Knowledge Enhancement Tool for Musculoskeletal Pathology. In: Shumaker, R. (ed.) Virtual and Mixed Reality, Part II, HCII 2011. LNCS, vol. 6774, pp. 54-63. Springer, Heidelberg (2011)

5. Stone, E.E., Skubic, M.: Passive In-Home Measurement of Stride-to-Stride Gait Variability Comparing Vision and Kinect Sensing. In: IEEE EMBS, Massachusetts USA (2011) 
6. Mitchell, G., Clarke, A.: Capturing and Visualising Playground Games and Performance: A Wii and Kinect based motion capture system. In: The Proceedings of the 2011 International Conference on Electronic Visualisation and the Arts (EVA 2011), pp. 218-225. British Computer Society, UK (2011)

7. Charissis, V., Ward, B.M., Naef, M., Rowley, D., Brady, L., Anderson, P.: An Enquiry into VR Interface Design for Medical Training: VR Augmented Anatomy Tutorials for Breast Cancer. In: McDowall, I.E., Dolinsky, M. (eds.) Proceedings of the: International Annual Symposium of IS\&T/SPIE, The Engineering Reality of Virtual Reality, San Jose, California, vol. 6804, pp. 19-28 (2008) ISBN: 9780819469762

8. Desloover, K., Wong, P., Swings, L., Callewaert, B., Vandenneucker, H., Leardini, A.: Range of motion and repeatability of knee kinematics for 11 clinically relevant motor tasks. Studies in Gait \& Posture 32, 597-602 (2010)

9. Foxlin, E., Harrington, M., Pfeifer, G.: Constellation: A wide range wireless motiontracking system for augmented Reality and Virtual set applications. In: Studies in Proceedings of Siggraph 1998 Computer Graphics Proceedings. ACM SIGGRAPH Conference Series (1998)

10. Zhou, H., Hu, H.: Human motion tracking for rehabilitation-A survey. Studies in Biomedical Signal Processing and Control, Elsevier LTD 3, 1-18 (2008); Perry, J., Burnfield, J.M.: Gait Cycle. In: Gait Analysis Normal and Pathological Function, vol. 2, pp. 3-19. Slack Incorporated (2010)

11. Noonan, D., Mountney, P., Elson, D., Darzi, Yang, G.: A Stereoscopic Fibroscope for Camera Motion and 3D Depth Recovery During Minimally Invasive Surgery. In: Proc. ICRA 2009, pp. 4463-4468 (2009)

12. Zöllner, M., Huber, S., Jetter, H., Reiterer, H.: NAVI - A Proof-of-Concept of a Mobile Navigational Aid for Visually Impaired Based on the Microsoft Kinect. Studies in University of Konstanz, Germany (2012)

13. Dutta, T.: Evaluation of the Kinect sensor for 3-D kinematic measurement in the workplace. Studies in Applied Ergonomics, 1-5 (2011), doi:10.1016

14. Stone, E.E., Skubic, M.: Passive In-Home Measurement of Stride-to-Stride Gait Variability Comparing Vision and Kinect Sensing. IEEE EMBS, Boston (2011)

15. Henry, P., et al.: RGB-D mapping: Using Kinect-style depth cameras for dense 3D modeling of indoor environments. The International Journal of Robotics Research, SAGE Journals (2012), doi:10.1177/0278364911434148

16. Izadi, S., et al.: KinectFusion: real-time 3D reconstruction and interaction using a moving depth camera. In: Proc. of 24th UIST annual ACM symposium on User Interface Software and Technology, pp. 559-568 (2011) 